\title{
Reduced $\gamma$-Aminobutyric Acid in Occipital and Anterior Cingulate Cortices in Primary Insomnia: a Link to Major Depressive Disorder?
}

\author{
David T Plante $^{*, 1}$, J Eric Jensen ${ }^{2,3}$, Laura Schoerning ${ }^{4}$ and John W Winkelman ${ }^{3,5}$ \\ 'Department of Psychiatry, University of Wisconsin School of Medicine and Public Health, Madison, WI, USA; ${ }^{2}$ Brain Imaging Center, \\ McLean Hospital, Belmont, MA, USA; ${ }^{3}$ Department of Psychiatry, Harvard Medical School, Boston, MA, USA; ${ }^{4}$ Sleep HealthCenters, \\ Brighton, MA, USA; ${ }^{5}$ Division of Sleep Medicine, Brigham and Women's Hospital, Boston, MA, USA
}

\begin{abstract}
Insomnia is closely related to major depressive disorder (MDD) both cross-sectionally and longitudinally, and as such, offers potential opportunities to refine our understanding of the neurobiology of both sleep and mood disorders. Clinical and basic science data suggest a role for reduced $\gamma$-aminobutyric acid (GABA) in both MDD and primary insomnia (PI). Here, we have utilized single-voxel proton magnetic spectroscopy (IH-MRS) at 4 Tesla to examine GABA relative to total creatine (GABA/Cr) in the occipital cortex (OC), anterior cingulate cortex (ACC), and thalamus in 20 non-medicated adults with PI ( 12 women) and 20 age- and sex-matched healthy sleeper comparison subjects. PI subjects had significantly lower GABA/Cr in the OC $(p=0.0005)$ and ACC $(p=0.03)$ compared with healthy sleepers. There was no significant difference in thalamic GABA/Cr between groups. After correction for multiple comparisons, GABA/Cr did not correlate significantly with insomnia severity measures among Pl subjects. This study is the first to demonstrate regional reductions of GABA in $\mathrm{PI}$ in the $\mathrm{OC}$ and $\mathrm{ACC}$. Reductions in GABA in similar brain regions in MDD using IH-MRS suggest a common reduction in cortical GABA among $\mathrm{PI}$ and mood disorders.

Neuropsychopharmacology (2012) 37, 1548-1557; doi:I0.1038/npp.20 I2.4; published online 8 February 2012
\end{abstract}

Keywords: insomnia; magnetic resonance spectroscopy; sleep disorders; GABA; occipital cortex; anterior cingulate cortex

\section{INTRODUCTION}

Insomnia is the most common sleep complaint in industrialized countries, affecting roughly one-third of all adults, and resulting in significant daytime consequences in approximately $5-10 \%$ of the adult population (Buysse, 2008). As a syndrome, insomnia is a significant public health problem associated with functional impairment, increased health care utilization, and disability (Simon and VonKorff, 1997). In prospective morbidity studies, insomnia is associated with an increased risk of developing major depressive disorder (MDD) (Breslau et al, 1996; Chang et al, 1997; Buysse et al, 2008; Szklo-Coxe et al, 2010). In the absence of an adequate understanding of its pathophysiology, insomnia is divided into primary and comorbid forms, the latter diagnosed when coexisting medical, sleep, or psychiatric disorders are present. Roughly $20-25 \%$ of those

*Correspondence: Dr DT Plante, Wisconsin Psychiatric Institute and Clinics, 600I Research Park Blvd., Madison, WI 537II, USA, Tel: + I (608) 232 33। I, Fax: + I (608) 231 90 I I, E-mail: dplante@wisc.edu Received 24 October 2011; revised 4 December 2011; accepted 3 January 2012 with chronic insomnia are considered to have primary insomnia (PI), in which insomnia occurs in the absence of a related medical or psychiatric illness (Buysse et al, 1994).

Gamma aminobutyric acid (GABA) is the primary inhibitory neurotransmitter in the central nervous system, and several lines of evidence suggest GABA has a role in the etiology and/or maintenance of insomnia. First, benzodiazepine receptor agonists (BZRAs), that are efficacious in the treatment of insomnia, promote sleep by increasing receptor affinity for GABA (Gottesmann, 2002). Second, physiological, neuroimaging, and cognitive investigations demonstrate hyperarousal in insomnia (Nofzinger et al, 2004; Bonnet and Arand, 2010; Riemann et al, 2010), which may suggest a relative decrement of inhibitory GABAergic neurotransmission (Klumpers et al, 2010). Finally, GABAergic nuclei in the brain, including the ventrolateral preoptic nucleus (VLPO) and thalamic reticular nucleus (TRN), have important roles in sleep initiation and maintenance. The VLPO promotes sleep via suppression of CNS arousal systems in the tuberomammillary nucleus and brainstem monoaminegic systems (Saper et al, 2005); the TRN acts as a gating mechanism between the thalamus and cortex, and has a key role in the generation of oscillatory activity 
during EEG-synchronized sleep (Steriade, 2003; Saper et al, 2005).

Preliminary work from our laboratory, using proton magnetic resonance spectroscopy (1H-MRS), demonstrated decreased global brain GABA in PI, the first neurochemical abnormality identified in this disorder (Winkelman et al, 2008). This study utilized a $2 \mathrm{D}$ chemical shift imaging (CSI) acquisition combined with J-resolved Echo-Planar Spectroscopic Imaging (JEPSI), which yielded a large amount of spectral information with a relatively short scan time. However, because GABA was summed over temporal, parietal, and occipital cortices, as well as subcortical structures including the thalamus and basal ganglia, concentrations of GABA in specific brain regions could not be determined. Thus, the primary aim of this study was to examine brain GABA using single-voxel $1 \mathrm{H}-\mathrm{MRS}$ at high field (4 Tesla) in specific regions of interest, utilizing J-difference editing using Point Resolved Spectroscopy with MEGA suppression (MEGAPRESS) (Mescher et al, 1998), which has evolved into the most commonly used and accurate spectroscopic method for quantifying GABA in vivo (Bogner et al, 2010; Henry et al, 2011). Our hypothesis was that GABA would be reduced in PI in the thalamus, occipital cortex (OC), and anterior cingulate cortex (ACC) relative to healthy controls. Thalamic reductions were hypothesized based on the role of the TRN in sleep (Steriade, 2003; Saper et al, 2005), animal and disease models in which damage to the thalamus induces insomnia (Villablanca and Salinas-Zeballos, 1972; Montagna, 2005), and prior spectroscopic studies demonstrating alterations in thalamic GABA from administration of BZRAs (Licata et al, 2009). Reductions in occipital GABA were based on anatomical overlap with preliminary 2D-CSI data (Winkelman et al, 2008); as well as the aforementioned relationship between insomnia and depression, with several 1H-MRS studies demonstrating decreased occipital GABA in MDD (Sanacora et al, 1999; Sanacora et al, 2004; Bhagwagar et al, 2007; Bhagwagar et al, 2008). Hypothesized reductions in GABA in the ACC were based on animal and human investigations of insomnia that demonstrate increased activity in the ACC (Nofzinger et al, 2004; Nofzinger et al, 2006; Cano et al, 2008), as well as 1H-MRS studies demonstrating decrements in GABA in prefrontal regions encompassing the ACC in MDD (Hasler et al, 2007; Bhagwagar et al, 2008; Price et al, 2009).

\section{MATERIALS AND METHODS}

\section{Participants}

Young adult and middle-aged (18-60 years) subjects were recruited from the greater Boston, MA area from July 2009 to January 2011. PI subjects met DSM-IV diagnostic criteria for PI, with a report of difficulty initiating or maintaining sleep or nonrestorative sleep with resulting daytime distress or dysfunction that was not attributable to another medical or psychiatric disorder. Consistent with recommended research diagnostic criteria (Buysse et al, 2006), additional severity criteria included a self-report of typical sleep onset latency (SOL) plus wake after sleep onset (WASO) of at least $30 \mathrm{~min}$, and a duration of insomnia $\geqslant 6$ months. Age and sex-matched healthy control subjects without sleep complaints were also recruited. Subjects were not permitted to use CNS active agents for 2 weeks prior to enrollment and for the duration of the study.

All subjects were evaluated with an unstructured clinical interview for history of sleep and medical disorders, and interview for lifetime history of psychiatric disorders with the Structured Clinical Interview for DSM-IV (SCID). Subjects were administered the Insomnia Severity Index (ISI) (Morin, 1993), Pittsburgh Sleep Quality Index (PSQI) (Buysse et al, 1989), Dysfunctional Beliefs and Attitudes about Sleep (DBAS-16) (Morin et al, 2007), and Beck Depression Inventory (BDI-IA) (Beck and Steer, 1993). Baseline laboratories included urine toxicology and pregnancy testing (for female subjects).

Exclusion criteria for all subjects included clinical evidence of any moderate to severe sleep disorder other than insomnia (eg obstructive sleep apnea, restless legs syndrome, etc.); current or past (within the preceding year) diagnosis of alcohol or drug dependence/abuse; history of significant medical or neurological illness including significant head trauma or loss of consciousness $>30 \mathrm{~min}$; BMI $>35 \mathrm{~kg} / \mathrm{m}^{2}$; consumption of $>10$ cigarettes per day, $>2$ caffeinated beverages per day, or $>2$ standard alcoholic drinks per day for a period $>1$ month within the preceding year; history of shift-work; peri-menopausal symptoms that disrupted sleep; contraindicated condition for MR scanning; and women who were pregnant, lactating, or planning to become pregnant during the study.

The study was approved by the Institutional Review Board of Partners Healthcare, the parent organization of Brigham and Women's Hospital and McLean Hospital, and carried out in accordance with the Declaration of Helsinki. All subjects received compensation for their participation in this study.

\section{Sleep Diaries and Actigraphy}

Following initial evaluation, subjects completed sleep-wake diaries supplemented by wrist-worn actigraphy (Actiwatch AW-64, Minimitter, Bend OR) for 2 weeks. Diaries included self-report of sleep-wake parameters (eg, bedtime and wake-time, estimated SOL, WASO, etc.), caffeine/alcohol/ medication consumption, and a visual analog scale (VAS) of subjective sleep quality. Subjects with PI were excluded if their sleep diaries did not demonstrate $\mathrm{SOL}+\mathrm{WASO}>30 \mathrm{~min}$ on the majority of days during the baseline period. To allow for inter-individual variation of total sleep time, but exclude subjects who were chronically sleep-restricted, sleeping excessive amounts of time, or had substantial night-to-night variation in sleep times, healthy sleeper controls were excluded if they demonstrated $<7.5$ or $>10$ hours of sleep on 10/14 nights during the screening period. Actigraphy corroborated sleep diaries, but was not used as an inclusionary/exclusionary measure per se.

\section{MR Imaging}

MR imaging was performed at approximately the same time of day (range 0900-1200 hours) and as soon as logistically possible after completion of baseline actigraphy/diaries (1-23 days). Subjects continued sleep diaries and actigraphy until the day of their scans to confirm that typical sleepwake patterns continued prior to MRS. Subjects were 
re-scanned if spectra were not obtained due to movement artifact or technical failure. Consistent with prior studies in MDD (Bhagwagar et al, 2008), female subjects were scanned during the follicular phase of their menstrual cycle to control for confounding hormonal effects on GABA.

Imaging and spectroscopy was performed on a whole body 4-Tesla MR scanner (Varian/UNITYInova, Palo Alto, CA) at McLean Hospital in Belmont, MA. Data collection utilized a birdcage-design, radio-frequency (RF) head coil operating at $170.3 \mathrm{MHz}$ for proton (XLR Imaging, London, Canada). Scout images confirmed optimal positioning, and unsuppressed water signal was shimmed to a global water linewidth of less than or equal to $25 \mathrm{~Hz}$. Subsequently, highcontrast $\mathrm{T}_{1}$-weighted anatomical images were taken in the sagittal and axial planes (echo time/repetition time $=6.2 \mathrm{~s} /$ $11.4 \mathrm{~ms}$, field-of-view $=24 \times 24 \times 8 \mathrm{~cm} \quad$ (sagittal) and $22 \times 22 \times 16 \mathrm{~cm}$ (axial), readout duration $=4 \mathrm{~ms}$, receive bandwidth $= \pm 32 \mathrm{kHz}$, in-plane matrix size $=128 \times 256 \times 16$ (sagittal) and $256 \times 256 \times 64$ (axial), in-plane resolution $=$ $0.94 \times 1.9 \mathrm{~mm}$ (sagittal) and $0.94 \times 0.94 \mathrm{~mm}$ (axial), readout points $=512$, slice thickness $=2.5 \mathrm{~mm}$, flip-angle $=11^{\circ}$ ) for anatomical landmarking and image segmentation analysis.

\section{Proton MRS}

The axial and sagittal high-resolution, $\mathrm{T}_{1}$-weighted anatomical images were used as a guide to systematically place single voxels in the left thalamic lobe (Thal) $(2 \times 3 \times 2 \mathrm{~cm})$, bilateral rostral anterior-cingulate cortex (ACC) $(3 \times 2 \times 2 \mathrm{~cm})$, and bilateral OC $(3 \times 2 \times 2 \mathrm{~cm})$ (Figure 1). Proton spectroscopy employed a GABA-optimized MEGAPRESS sequence (Mescher et al, 1998) for optimal measures of GABA using the difference-editing technique, as well as secondary measures of glutamate (Glu), N-acetylaspartate (NAA), total creatine $(\mathrm{Cr})$ and total choline (Cho) in the $68 \mathrm{~ms}$ subspectrum. Manual shimming of the magnetic field within each prescribed voxel achieved water linewidths ranging from $7-12 \mathrm{~Hz}$. Following the automated optimization of water suppression power and tip angles, the transmitter frequency was set onto the creatine resonance at $3.00 \mathrm{ppm}$ to minimize chemical-shift displacement artifact for each spectral acquisition. The MEGAPRESS sequence used the following acquisition parameters: $\mathrm{TR}=2 \mathrm{~s}, \mathrm{TE}=68 \mathrm{~ms}$, spectral bandwidth $=2 \mathrm{kHz}$, readout duration $=512 \mathrm{~ms}$, $\mathrm{NEX}=384$, total scan duration $=13 \mathrm{~min}$.

\section{Proton MRS Processing}

All spectroscopic data processing and analyses were undertaken on a LINUX workstation using reconstruction code written on-site (C-code) and commercial fitting software. In order to quantify difference-edited GABA with MEGAPRESS data, the difference-edited spectra were fitted with LCModel (Provencher, 1993; Provencher, 2001) using basis sets acquired from phantoms at $4 \mathrm{~T}$. All phase and frequency-corrected 'ON' and 'OFF' $68 \mathrm{~ms}$ sub-spectra were then averaged separately to produce a single $68 \mathrm{~ms}$ ' $\mathrm{ON}$ ' and 'OFF' spectrum, which were then subsequently subtracted to produce the final, optimized, difference-edited GABA spectrum. The appropriate phantom-based LCModel templates were used to fit the $68 \mathrm{~ms}$ 'OFF' spectrum for the

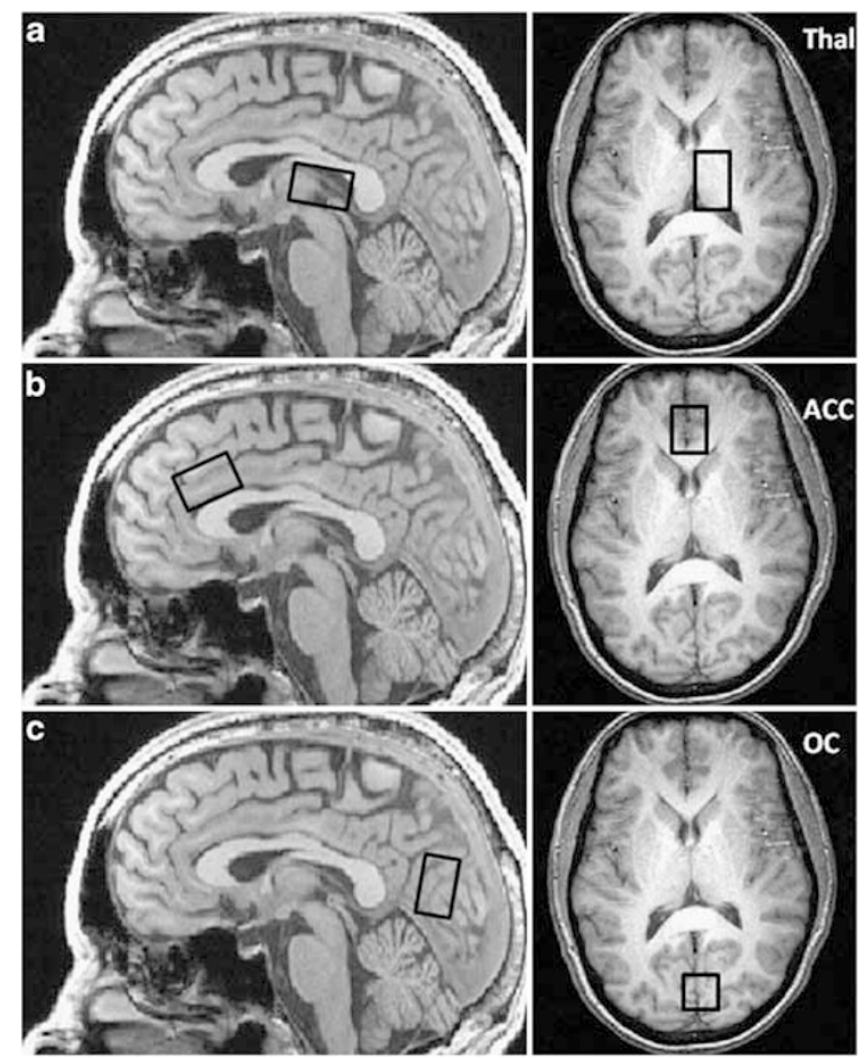

Figure I Anatomical placement for thalamic (a), anterior cingulate cortex (b) and occipital cortex (c) proton magnetic spectroscopy $(\mathrm{IH}-$ MRS) voxels.

measurement of creatine, glutamate and other metabolites (Figure 2). The difference-edited GABA resonance area at $3.00 \mathrm{ppm}$, as well as the $68 \mathrm{~ms}$ 'OFF' spectrum metabolite areas, were normalized to the LCModel fitted $68 \mathrm{~ms}$ 'OFF' spectrum creatine resonance area and left as simple ratios.

\section{Image Segmentation}

To ascertain gray and white matter contribution to each voxel, the axial T1-weighted images were segmented into gray matter, white matter, and cerebrospinal fluid compartments using FSL version 4.1 (FMRIB Software Library; Analysis Group, FMRIB; Oxford, UK) in combination with an in-house automated voxel co-registration and partialvolume analysis program.

\section{Statistics}

The a priori primary outcome variable was the difference in GABA/Cr between PI and controls in each region of interest, tested with unpaired t-test (two-tailed). Based on previous estimates of global brain GABA/Cr in PI (Winkelman et al, 2008), we estimated $80 \%$ power to detect a difference between groups at $\alpha=0.05$, two-sided with $n=20$ in each group. Voxels were considered outliers if $\mathrm{Cr}$ fell outside two standard deviations from the mean for all subjects. GABA/ $\mathrm{Cr}$ data for individual subjects were excluded if LCModel was unable to fit GABA resonance. Voxel tissue composition, demographics, psychometric scores, and diary/actigraphic 


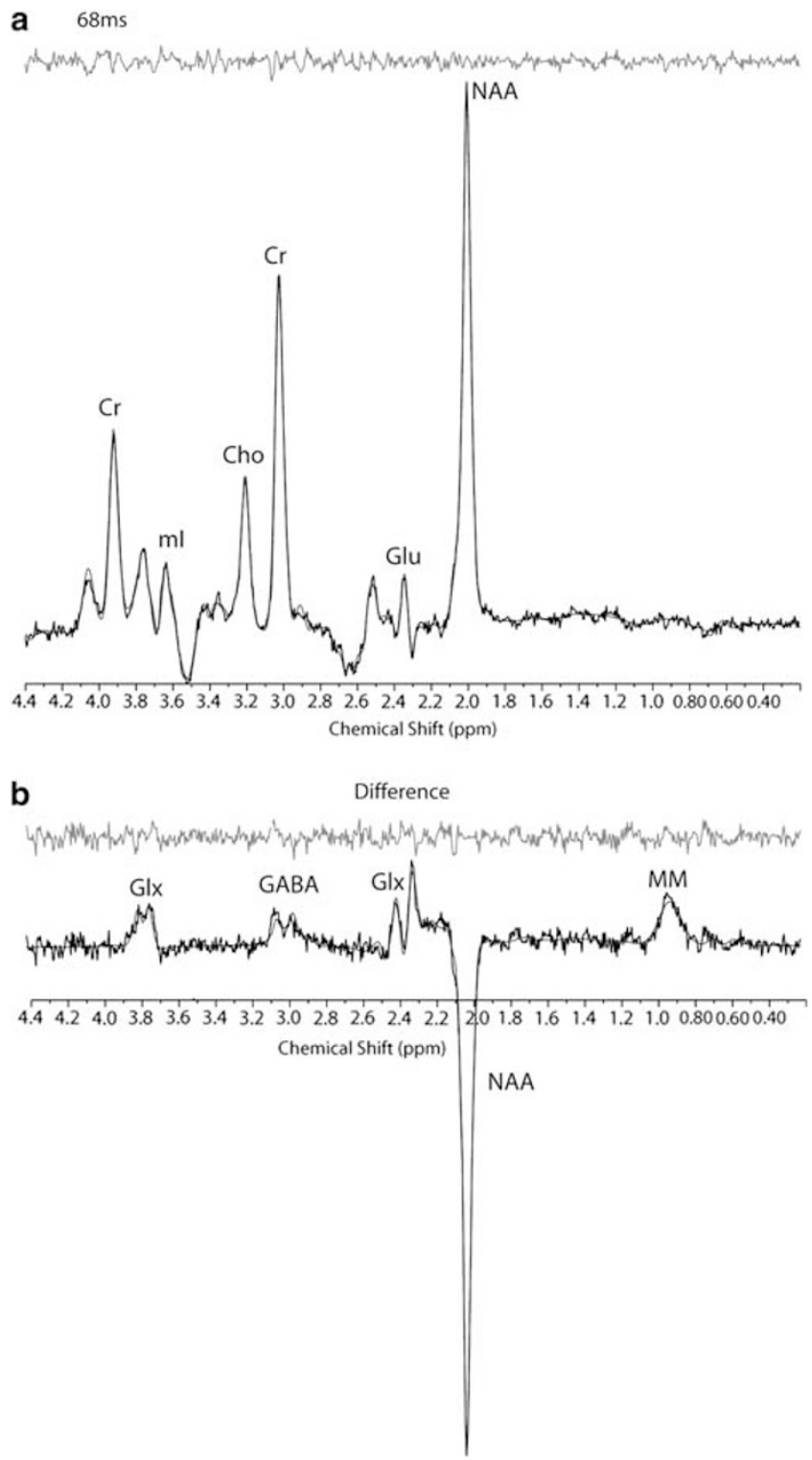

Figure 2 Point Resolved Spectroscopy with MEGA suppression (MEGAPRESS) $68 \mathrm{~ms}$ sub-spectrum (a) and $\gamma$-aminobutyric acid (GABA) difference-edited spectrum (b) from the occipital cortex (OC) of a study subject. All spectra are displayed with no filtering and show the LCModel fit and residual. Metabolites include: total creatine $(\mathrm{Cr})$, myo-inositol ( $\mathrm{ml}$ ), total choline (Cho), N-acetylaspartate (NAA), glutamate (Glu), glutamine plus glutamate $(\mathrm{G} \mid \mathrm{x}), \quad \gamma$-aminobutyric acid (GABA), and co-edited macromolecule resonance at $\sim 0.9 \mathrm{ppm}(\mathrm{MM})$.

data between groups were compared using unpaired t-tests (two-sided). Differences in other metabolites (NAA/Cr, Cho/Cr, and $\mathrm{Glu} / \mathrm{Cr}$ ) between groups, and Pearson's correlations for insomnia severity measures versus GABA/ Cr were examined on an exploratory basis, with Bonferroni correction for multiple comparisons. Statistical analysis was performed using JMP 9 (SAS, Cary, NC).

\section{RESULTS}

The PI group $(n=20)$ was comprised of 12 women, with a mean age of $34.3 \pm 8.3$ (range 22-53) years; the age and sex-matched control group had a mean age of $34.1 \pm 9.9$ (range 23-55) years (Table 1). All PI subjects had a continuous history of insomnia for at least 1 year, with $16 / 20$ reporting duration of insomnia $\geqslant 5$ years (mean 10.1 years). No PI subject had a prior lifetime history of mood or psychotic disorder; one PI subject had a prior history of anxiety disorder not otherwise specified that had been in remission $>6$ months. Nine PI subjects reported lifetime history of sedative-hypnotic use (4 BZRAs; 5 non-GABAergic hypnotics); five of whom reported non-nightly use within 1 month of enrollment (2 BZRAs; 3 non-GABAergic hypnotics), with the remaining subjects reporting no use of sedative-hypnotics for at least 4 months. Psychometric scales, sleep-wake diaries, and actigraphy corroborated their insomnia complaint (Table 1). BDI scores were significantly higher in PI versus controls, though still far below standard cutoffs for a clinical mood disorder and consistent with typical daytime consequences of insomnia (eg, irritability, fatigue). One male PI subject did not complete the PSQI; two subjects ( 1 female PI and 1 female control) did not complete the BDI. Baseline actigraphic data for one male PI subject was not available due to device failure.

Two PI subjects required repeat MRS scans due to movement artifact. Voxels excluded from analysis due to $\mathrm{Cr}$ outliers were 2 Thal $(\mathrm{PI}=1 \mathrm{male}$, control $=1 \mathrm{male}), 2 \mathrm{OC}$ $(\mathrm{PI}=1$ female, control=1 male), and 1 ACC $(\mathrm{PI}=1$ female). One voxel in the ACC (female PI) and two voxels in the OC ( 2 female controls) were excluded from $\mathrm{GABA} / \mathrm{Cr}$ analysis due to failure of LCModel to fit the GABA resonance.

PI subjects had significantly reduced $\mathrm{GABA} / \mathrm{Cr}$ levels compared with controls in the OC $(\mathrm{PI}=0.18 \pm 0.06$; controls $=0.27 \pm 0.07, \mathrm{df}=34, \mathrm{t}=-3.83, p=0.0005)$ and ACC $\quad(\mathrm{PI}=0.15 \pm 0.05 ; \quad$ controls $=0.19 \pm 0.05, \quad \mathrm{df}=36$, $t=-2.32, p=0.03$ ) (Table 2; Figure 3). Post-hoc analysis to examine possible effects of medications and duration of insomnia revealed that exclusion of PI subjects who had taken sedative-hypnotics (GABAergic or otherwise) within 1 month of enrollment did not substantively alter findings in either the OC $(\mathrm{PI}=0.17 \pm 0.06$; controls $=0.27 \pm 0.07, \mathrm{df}=29$, $t=-3.95, p=0.0005)$ or ACC $(\mathrm{PI}=0.15 \pm 0.06$; controls $=$ $0.19 \pm 0.05, \mathrm{df}=32, t=-2.01, p=0.05)$. Similarly, exclusion of subjects whose insomnia was $<5$ years duration did not alter findings in the $\mathrm{OC}(\mathrm{PI}=0.18 \pm 0.07$; controls $=0.27 \pm 0.07, \mathrm{df}=30, t=-3.21, p=0.003)$ or ACC $(\mathrm{PI}=0.15 \pm 0.06$; controls $=0.19 \pm 0.05, \mathrm{df}=33, t=-2.06$, $p=0.05)$. In addition, due to prior MRS studies that have demonstrated increases in occipital GABA in the follicular phase in healthy women relative to both men and women with premenstrual dysphoric disorder (Epperson et al, 2002; Epperson et al, 2005), and aforementioned exclusion of data resulting in loss of sex-matching in the OC and ACC, additional analyses were performed to investigate the effects of sex. Two-way ANOVA demonstrated a significant effect of diagnosis in both the $\mathrm{OC}\left(\mathrm{F}_{1,32}=15.23, p=0.0005\right)$ and ACC $\left(\mathrm{F}_{1,34}=5.90, p=0.02\right)$, without a significant effect of sex (OC: $\mathrm{F}_{1,32}=0.08, p=0.78$; ACC: $\mathrm{F}_{1,34}=0.05, p=0.83$ ) or diagnosis $\mathrm{x}$ sex interaction (OC: $\mathrm{F}_{1,32}=1.09, p=0.30$; ACC: $\left.\mathrm{F}_{1,34}=0.82, p=0.37\right)$ in either region.

There was no significant difference in $\mathrm{GABA} / \mathrm{Cr}$ between PI and controls in the thalamus (PI $=0.24 \pm 0.07$; controls $=0.25 \pm 0.05, \mathrm{df}=36, t=-0.71, p=0.48) \quad$ (Table 2; 
Table I Demographics and Sleep-wake Measures for Primary Insomnia and Controls

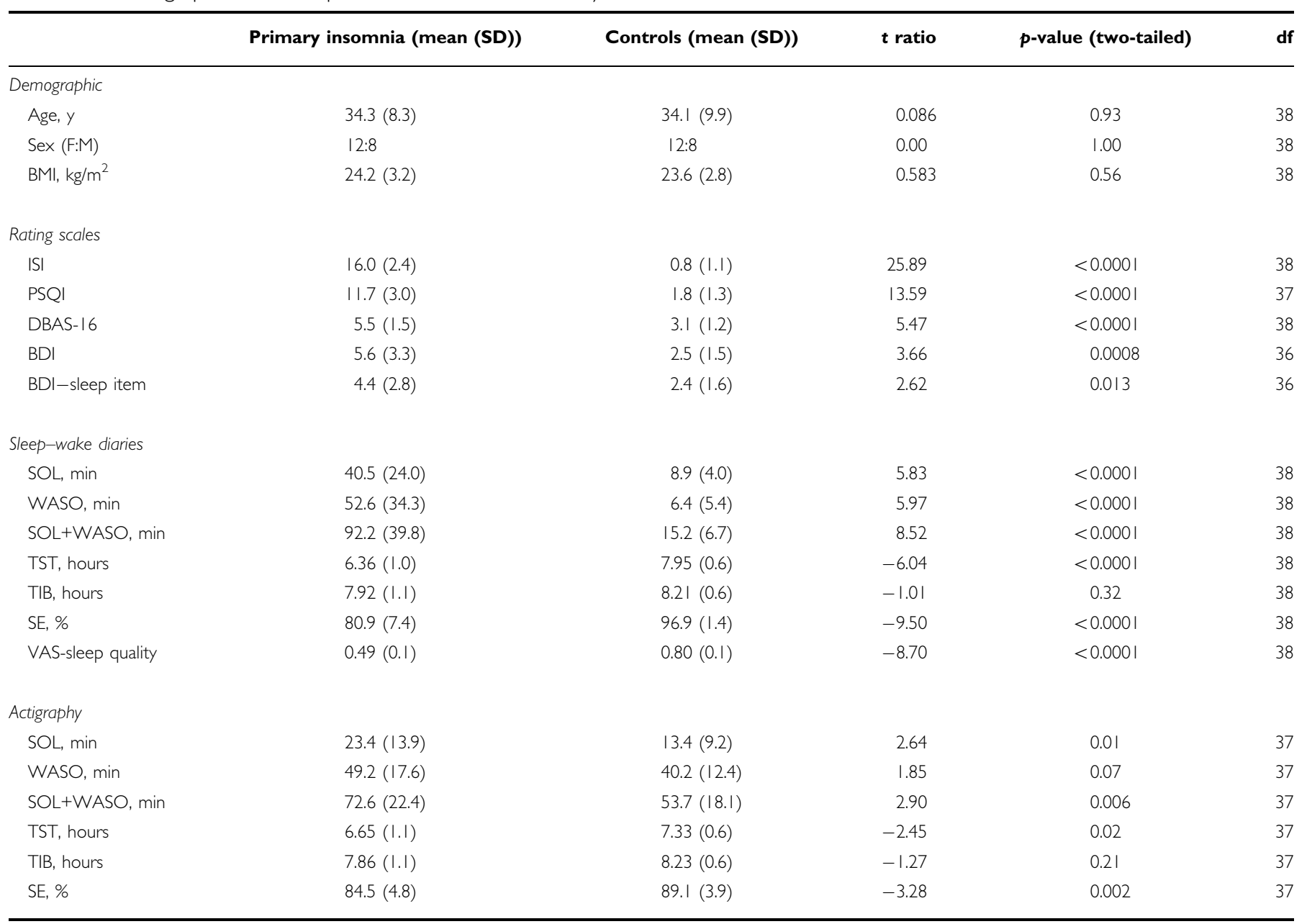

Abbreviations: BMI, body mass index; ISI, Insomnia Severity Index; PSQI, Pittsburgh Sleep Quality Index; DBAS- I6, Dysfunctional Beliefs and Attitudes about Sleepsixteen Item; BDI, Beck Depression Inventory IA; BDI-sleep item, BDI score minus sleep-related items; SOL, sleep onset latency; WASO, wake after sleep onset; TST, total sleep time; TIB, time in bed; SE, sleep efficiency; VAS-sleep quality, visual analog scale of sleep quality.

Figure 3). There were no significant differences between PI and controls for $\mathrm{Cr}, \% \mathrm{GM}$, or \%WM in any region of interest (Table 2). Exploratory analyses revealed no significant differences in levels of other metabolites between PI and controls after correcting for multiple comparisons (Table 2). Pearson's correlations of sleep measures and GABA in PI subjects demonstrated a correlation between total sleep time (as measured by actigraphy) and GABA in the ACC $(r=0.51, p=0.03)$, however this correlation did not remain significant after correcting for multiple comparisons.

\section{DISCUSSION}

Our findings of reduced brain GABA in unmedicated PI in the OC and ACC, by $33 \%$ and $21 \%$, respectively, confirms preliminary data from our laboratory and identifies specific brain regions in which GABA is diminished in PI. These findings provide important insights into the neurobiology of insomnia, and validate the theory of altered GABAergic neurotransmission in PI, which has been suggested by circumstantial anatomical, neurophysiological, and pharmacological evidence, as well as preliminary data from our laboratory (Winkelman et al, 2008). Although the mechanisms through which reduced GABA in the ACC and OC relate to the pathophysiology of insomnia are unknown, it is noteworthy that increased activity in the ACC has been implicated in animal models of insomnia (Cano et al, 2008) as well as human $\left({ }^{18} \mathrm{FDG}-\mathrm{PET}\right)$ investigations of PI (Nofzinger et al, 2004; Nofzinger et al, 2006). Furthermore, altered regional cerebral blood flow (rCBF) in PI as measured by single photon emission computed tomography (SPECT) has been demonstrated during NREM sleep across a number of brain regions, including frontal and occipital cortices (Smith et al, 2002), and rCBF in the ACC during wakefulness correlates with insomnia severity in MDD (Périco et al, 2005). Although it is not currently clear how MRS-derived measures of GABA directly relate to synaptic activity or cortical excitability, our results are congruent with the hyperarousal model of PI, in which chronic insomnia is due to increased arousal across a range of cognitive and physiological domains, which may be consistent with an imbalance between excitatory and inhibitory neurotransmission in multiple cortical regions (Bonnet and Arand, 2010; Riemann et al, 2010).

Beyond the implications for neurobiological research in PI, the findings of this study may have potential implications 
Table 2 IH-MRS Measures in Primary Insomnia versus Controls

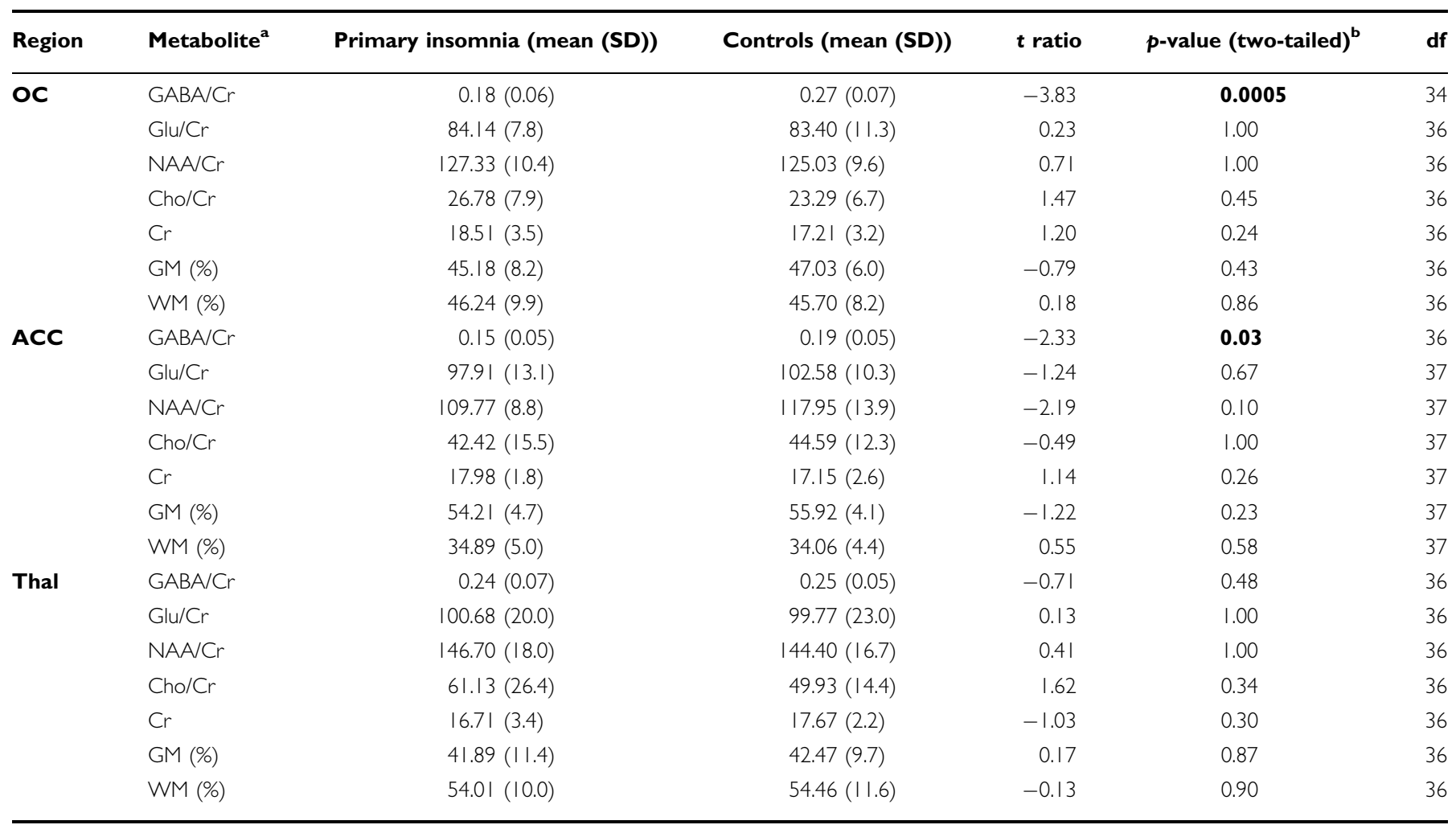

aMetabolites/Cr reported $\times 100$. Glu/Cr, NAA/Cr, and Cho/Cr Bonferroni corrected for multiple comparisons.

balues in bold denote significant differences between gruoups.

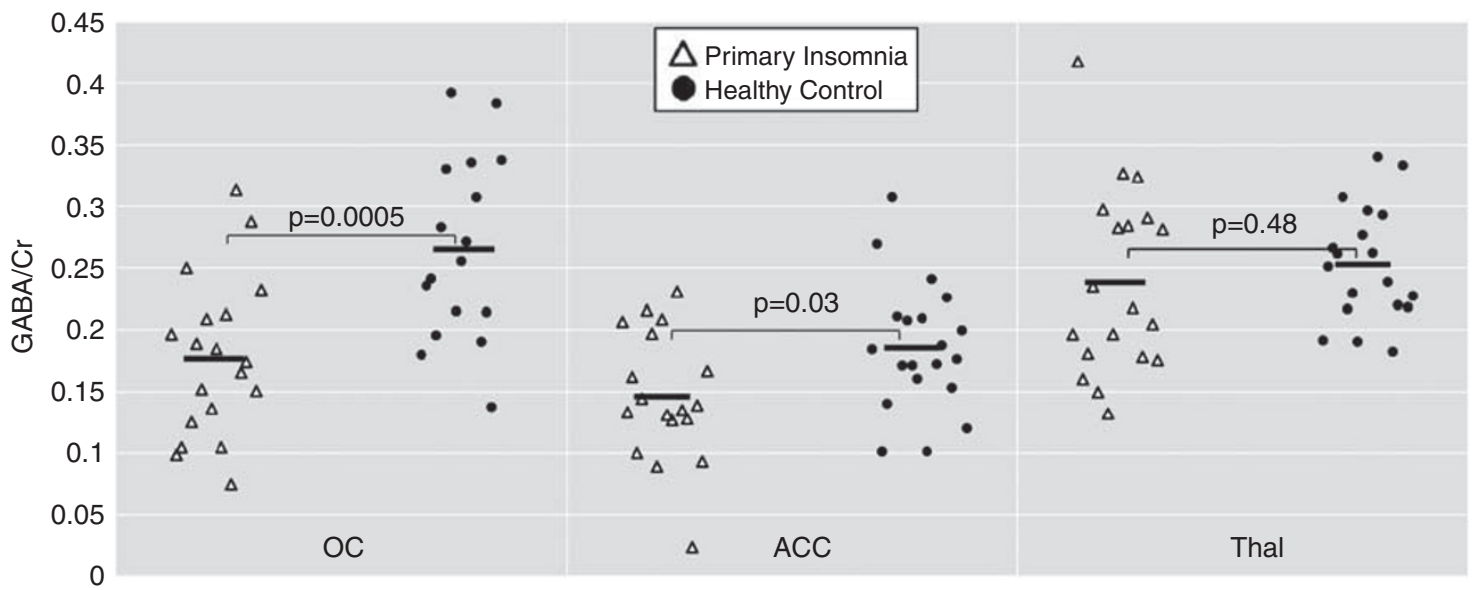

Figure 3 Individual subject GABA/Cr by region of interest. Solid bars denote group means.

for research regarding GABAergic alterations in mood disorders. Over the last 30 years, there has been growing evidence that MDD is associated with altered GABAergic neurotransmission (Sanacora, 2010; Luscher et al, 2011). MDD patients have demonstrated low plasma and CSF GABA (Gold et al, 1980; Petty and Schlesser, 1981), decreased cell density of GABAergic neurons in both the OC and prefrontal regions in autopsy studies (Rajkowska et al, 2007; Maciag et al, 2010), decrements of the GABA synthesizing enzyme glutamic acid decarboxylase in the dorsolateral prefrontal cortex (Gos et al, 2009; Karolewicz et al, 2010), and impaired measures of cortical inhibition using transcranial magnetic stimulation (TMS), consistent with decrements in GABAergic tone (Bajbouj et al, 2006; Lefaucheur et al, 2008; Levinson et al, 2010). However, the most influential data suggesting impaired GABAergic neurotransmission in MDD comes from $1 \mathrm{H}-\mathrm{MRS}$ studies that directly measure brain GABA in vivo. $1 \mathrm{H}-\mathrm{MRS}$ investigations in MDD demonstrate decrements of GABA in prefrontal regions (in voxels containing the ACC) and the OC (Sanacora et al, 1999; Sanacora et al, 2004; Hasler et al, 2007; Price et al, 2009) of similar magnitude to those observed in this study of PI, indicating that decreased GABA in the OC and ACC is a shared neurobiological finding in these disorders, and 
although speculative, suggests a physiological connection between the well-established epidemiological relationship between insomnia and MDD (Breslau et al, 1996; Chang et al, 1997; Ohayon and Roth, 2003; Buysse et al, 2008; Szklo-Coxe et al, 2010; Baglioni et al, 2011).

Insomnia is a risk factor for incident MDD, increasing the risk of depressive illness roughly twofold compared with those without sleep difficulties (Baglioni et al, 2011). However, the majority of individuals with insomnia do not go on to develop depression (Chang et al, 1997), suggesting PI is not simply a trait-marker for MDD. To develop effective strategies that would prevent the future development of MDD among individuals with PI requires a deeper understanding of the biological factors that convey cumulative risk for the development of depression. To account for heterogeneity among psychiatric disorders, the use of endophenotypes, which are more elementary, state-independent, intermediate phenotypes that bridge genetic variation to complex disease states, is a promising line of research (Gottesman and Gould, 2003). It has been posited that reduced cortical GABA is the most promising imaging endophenotype in MDD (Hasler and Northoff, 2011). However, 1H-MRS measures of GABA do not correlate with depression severity (Sanacora et al, 2004; Hasler et al, 2007; Price et al, 2009) and reductions in brain GABA in the OC have been demonstrated in panic disorder (Goddard et al, 2001) and alcohol dependence (Behar et al, 1999), suggesting that reduced GABA as an endophenotype is not specific for MDD. However, it is noteworthy that, in addition to increasing risk for incident depression, insomnia increases the risk for, and is a common comorbid symptom of, anxiety and substance use disorders (Ford and Kamerow, 1989; Breslau et al, 1996; Weissman et al, 1997; Neckelmann et al, 2007). Thus, although speculative, it is possible that insomnia may be an important unmeasured covariate in these studies, which could account for reductions observed in cortical GABA in multiple disorders.

The notion that comorbid insomnia is an important consideration in 1H-MRS investigations of GABA in psychiatric disorders is further supported by the fact that atypical depression, which is frequently associated with hypersomnia rather than insomnia, is not associated with reductions in GABA (Sanacora et al, 2004). In addition, the literature on reduced cortical GABA as a trait-marker for MDD is inconsistent, with increased, normal, and persistently lowered GABA in remitted MDD subjects reported (Sanacora et al, 2003; Hasler et al, 2005; Bhagwagar et al, 2007; Bhagwagar et al, 2008). Because residual insomnia is a common, but not universal symptom among remitted depressed subjects (Nierenberg et al, 2010), it is plausible that failure to account for the effects of comorbid insomnia as part of study design may have affected findings in these investigations.

The findings of this study and the aforementioned literature underscore the need for further research to clarify the relationships between clinical sleep disturbance and brain GABA. For PI research, studies utilizing other means to probe GABAergic neurotransmission (eg CSF, autopsy studies) that have already been applied in MDD, could help clarify the role of GABA in the pathophysiology of PI. Similarly, the relationship of brain GABA to other established physiological and cognitive markers of hyperarousal in insomnia may provide deeper insights into that conceptual framework. In addition, future studies that critically examine the role of comorbid sleep disturbance (eg insomnia and/or hypersomnia) in mood disorders and GABAergic neurotransmission are required to clarify whether alterations in GABA are a biomarker for a given mental illness, or an epiphenomenon related to sleep disturbance. Finally, $1 \mathrm{H}-$ MRS investigations in both PI and MDD that examine GABA in regions other than occipital and prefrontal cortices and utilize longitudinal designs may help distinguish regional patterns of alterations in GABA that segregate these disorders and clarify whether reduced cortical GABA is a trait-marker for insomnia and/or depressive illness.

There are limitations of this study that merit discussion. Most notably, polysomnography (PSG) was not performed, and thus, correlations between PSG measures of insomnia severity and GABA that have been demonstrated in prior studies cannot be corroborated (Winkelman et al, 2008). In addition, subjects in either group may have potentially had occult sleep disorders other than PI that may have affected results. However, PSG is not indicated for the routine clinical evaluation of insomnia, (Littner et al, 2003) and has not been used in 1H-MRS studies of MDD, even though sleep-related breathing and movement disorders are frequently associated with psychiatric symptoms (Beebe and Gozal 2002; Winkelman et al, 2006). The possibility that occult sleep disorders may have affected results was also minimized by thorough sleep and psychiatric history performed by psychiatrists with fellowship training in sleep medicine (DTP and JWW).

In addition, our findings of reduced GABA in the ACC in PI are not universally comparable to prior MDD $1 \mathrm{H}-\mathrm{MRS}$ studies that have examined the ACC. Positioning of our voxel in rostral ACC more closely approximates previous studies that have demonstrated significant GABA reductions in MDD in dorsomedial/dorsolateral prefrontal regions (Hasler et al, 2007; Bhagwagar et al, 2008), and is not directly comparable to voxels positioned in the pregenual/ventromedial prefrontal cortex utilized in other investigations (Hasler et al, 2007; Price et al, 2009). Moreover, although a recent study has demonstrated the diurnal stability of MEGAPRESS in quantification of brain GABA in healthy individuals (Evans et al, 2010), because of recent evidence suggesting the timing of $1 \mathrm{H}$-MRS is important in the study of cortical GABA in shift-work (Kakeda et al, 2011), it is plausible that results of this study may have been different had MRS scans been performed at different times of a day. Also, without behavioral or EEG monitoring, it is possible that differences between groups may have been due to either systematic differences in sleep-wake states and/or changes in visual stimulation due to eyes being open or closed at the time of $1 \mathrm{H}$-MRS acquisition. However, it is noteworthy that despite disrupted nocturnal sleep, PI subjects are not more likely to fall asleep during multiple sleep latency testing compared with healthy controls (Edinger et al, 2008), and recent data suggest GABA is not altered by visual stimulation in the OC (Lin et al, 2011). Finally, this study is not able to determine whether decrements in cortical GABA observed are due to PI itself, or result from chronic partial sleep deprivation associated with the illness. 
There are also inherent limitations to MRS that may affect interpretation of this study. First, differences in tissue composition (\%GM) or the concentration of an internal standard (Cr) may affect group differences in $\mathrm{GABA} / \mathrm{Cr}$ (Geramita et al, 2011). However, there were no differences between groups in tissue composition or $\mathrm{Cr}$ for any voxel, suggesting results are due to differences in GABA concentration, rather than other confounding factors. Additionally, MRS has limited spatial and temporal resolution compared with other neuroimaging modalities, and therefore, only the aggregate quantity of GABA in a relatively large brain region can be measured using $1 \mathrm{H}-\mathrm{MRS}$. This may be particularly relevant to our negative findings in the thalamus, as the TRN, which is involved in sleep initiation and maintenance, encapsulates several other nuclei and comprises a relatively small portion of the volume of the thalamus. In addition, our results cannot clarify whether decreased GABA in the ACC or OC in PI reflect decrements in GABAergic cell number or reduced quantity/production in an intact population of neurons. Finally, single voxel 1H-MRS requires the a priori selection of specific brain areas of interest, and thus levels of GABA in PI in other brain regions not examined in this study remain unknown.

In summary, our study provides the first evidence of reduced brain GABA in the occipital and anterior cingulate cortices of those with PI compared with healthy sleeper controls. Similar findings of reduced GABA in these brain regions in MDD suggest a common neurochemical alteration in these disorders. Given the strong cross-sectional and longitudinal epidemiological links between insomnia and MDD, further research that examines GABAergic neurotransmission both in PI and insomnia comorbid with MDD may shed further light on the connections between insomnia and mental illness.

\section{ACKNOWLEDGEMENTS}

This work was supported in part by the American Sleep Medicine Foundation Physician Scientist Training Award to DTP (\#48-PA-09). The funding organizations had no role in the design and conduct of the study; collection, management, analysis, and interpretation of the data; or preparation, review, or approval of the manuscript. This work was presented at the meeting of the Associated Professional Sleep Societies, Minneapolis, MN, June 11-15, 2011.

\section{DISCLOSURE}

Dr Plante reports having owned stock in Pfizer, and having received honoraria from Oakstone Medical Publishing and royalties from Cambridge University Press. Dr Winkelman reports serving as a consultant or advisory board member for Axon Laboratories, Boehringer-Ingelheim, Covance, GlaxoSmithKline, Impax Laboratories, Jazz Pharmaceuticals, Luitpold Pharmaceuticals, Neurogen, Novadel Pharma, Pfizer, Sunovion, Takeda, UCB, and Zeo; research support from Boehringer-Ingelheim, UCB (Schwarz) Pharma, GlaxoSmithKline, and Sepracor; and stock options in Axon Laboratories. Dr Jensen and Ms Schoerning report no conflict of interest.

\section{REFERENCES}

Baglioni C, Battagliese G, Feige B, Spiegelhalder K, Nissen C, Voderholzer $\mathrm{U}$ et al (2011). Insomnia as a predictor of depression: A meta-analytic evaluation of longitudinal epidemiological studies. J Affect Disord 135: 10-19.

Bajbouj M, Lisanby SH, Lang UE, Danker-Hopfe H, Heuser I, Neu $P$ (2006). Evidence for impaired cortical inhibition in patients with unipolar major depression. Biol Psychiatry 59: 395-400.

Beck AT, Steer RA (1993). Manual for the Beck Depression Inventory. Psychological Corporation: San Antonio, TX.

Behar KL, Rothman DL, Petersen KF, Hooten M, Delaney R, Petroff OA et al (1999). Preliminary evidence of low cortical GABA levels in localized $1 \mathrm{H}-\mathrm{MR}$ spectra of alcohol-dependent and hepatic encephalopathy patients. Am J Psychiatry 156: 952-954.

Beebe DW, Gozal D (2002). Obstructive sleep apnea and the prefrontal cortex: towards a comprehensive model linking nocturnal upper airway obstruction to daytime cognitive and behavioral deficits. J Sleep Res 11: 1-16.

Bhagwagar Z, Wylezinska M, Jezzard P, Evans J, Ashworth F, Sule A et al (2007). Reduction in occipital cortex gamma-aminobutyric acid concentrations in medication free recovered unipolar depressed and bipolar subjects. Biol Psychiatry 61: 806-812.

Bhagwagar Z, Wylezinska M, Jezzard P, Evans J, Boorman E, Matthews PM et al (2008). Low GABA concentrations in occipital cortex and anterior cingulate cortex in medication-free, recovered depressed patients. Int J Neuropsychopharmacol 11: 255-260.

Bogner W, Gruber S, Doelken M, Stadlbauer A, Ganslandt O, Boettcher $\mathrm{U}$ et al (2010). In vivo quantification of intracerebral GABA by single-voxel (1)H-MRS-How reproducible are the results? Eur J Radiol 73: 526-531.

Bonnet MH, Arand DL (2010). Hyperarousal and insomnia: state of the science. Sleep Med Rev 14: 9-15.

Breslau N, Roth T, Rosenthal L, Andreski P (1996). Sleep disturbance and psychiatric disorders: a longitudinal epidemiological study of young adults. Biol Psychiatry 39: 411-418.

Buysse DJ (2008). Chronic insomnia. Am J Psychiatry 165: 678-686. Buysse DJ, Ancoli-Israel S, Edinger JD, Lichstein KL, Morin CM (2006). Recommendations for a standard research assessment of insomnia. Sleep 29: 1155-1173.

Buysse DJ, Angst J, Gamma A, Ajdacic V, Eich D, Rossler W (2008). Prevalence, course, and comorbidity of insomnia and depression in young adults. Sleep 31: 473-480.

Buysse DJ, Reynolds III CF, Monk TH, Berman SR, Kupfer DJ (1989). The Pittsburgh Sleep Quality Index: a new instrument for psychiatric practice and research. Psychiatry Res 28: 193-213.

Buysse DJ, Reynolds III CF, Hauri PJ, Roth T, Stepanski EJ, Thorpy MJ et al (1994). Diagnostic concordance for DSM-IV sleep disorders: a report from the APA/NIMH DSM-IV field trial. Am J Psychiatry 151: 1351-1360.

Cano G, Mochizuki T, Saper CB (2008). Neural circuitry of stressinduced insomnia in rats. J Neurosci 28: 10167-10184.

Chang PP, Ford DE, Mead LA, Cooper-Patrick L, Klag MJ (1997). Insomnia in young men and subsequent depression. The Johns Hopkins Precursors Study. Am J Epidemiol 146: 105-114.

Edinger JD, Means MK, Carney CE, Krystal AD (2008). Psychomotor performance deficits and their relation to prior nights' sleep among individuals with primary insomnia. Sleep 31: 599-607.

Epperson CN, Haga K, Mason GF, Sellers E, Gueorguieva R, Zhang $\mathrm{W}$ et al (2002). Cortical $\gamma$-aminobutyric acid levels across the menstrual cycle in healthy women and those with premenstrual dysphoric disorder A 1H-MRS study. Arch Gen Psychiatry 59: 851-858.

Epperson CN, O’Malley S, Czarkowski KA, Gueorguieva R, Jatlow P, Sanacora G et al (2005). Sex, GABA, and nicotine: the impact of smoking on cortical GABA levels across the menstrual cycle as measured with proton magnetic resonance spectroscopy. Biol Psychiatry 57: 44-48. 
Evans CJ, McGonigle DJ, Edden RA (2010). Diurnal stability of $\gamma$ aminobutyric acid concentration in visual and sensorimotor cortex. J Magn Reson Imaging 31: 204-209.

Ford DE, Kamerow DB (1989). Epidemiologic study of sleep disturbance and psychiatric disorders: an opportunity for prevention? JAMA 262: 1479-1484.

Geramita M, Van der Veen JW, Barnett AS, Savostyanova AA, Shen J, Weinberger DR et al (2011). Reproducibility of prefrontal yaminobutyric acid measurements with J-edited spectroscopy. NMR Biomed 24: 1089-1098.

Goddard AW, Mason GF, Almai A, Rothman DL, Behar KL, Petroff OA et al (2001). Reductions in occipital cortex GABA levels in panic disorder detected with 1h-magnetic resonance spectroscopy. Arch Gen Psychiatry 58: 556-561.

Gold B, Bowers MB, Roth RH, Sweeney DW (1980). GABA levels in CSF of patients with psychiatric disorders. Am J Psychiatry 137: 362-364.

Gos T, Günther K, Bielau H, Dobrowolny H, Mawrin C, Trübner K et al (2009). Suicide and depression in the quantitative analysis of glutamic acid decarboxylase-immunorective neuropil. J Affect Disord 113: 45-55.

Gottesman II, Gould TD (2003). The endophenotype concept in psychiatry: etymology and strategic intentions. Am J Psychiatry 160: 636-645.

Gottesmann C (2002). GABA mechanisms and sleep. Neuroscience 111: 231-239.

Hasler G, Neumeister A, van der Veen JW, Tumonis T, Bain EE, Shen $\mathrm{J}$ et al (2005). Normal prefrontal gamma-aminobutyric acid levels in remitted depressed subjects determined by proton magnetic resonance spectroscopy. Biol Psychiatry 58: 969-973.

Hasler G, Northoff G (2011). Discovering imaging endophenotypes for major depression. Mol Psychiatry 16: 604-619.

Hasler G, van der Veen JW, Tumonis T, Meyers N, Shen J, Drevets WC (2007). Reduced prefrontal glutamate/glutamine and gamma-aminobutyric acid levels in major depression determined using proton magnetic resonance spectroscopy. Arch Gen Psychiatry 64: 193-200.

Henry ME, Lauriat T, Shanahan M, Renshaw PF, Jensen JE (2011). Accuracy and stability of measuring GABA, glutamate, and glutamine by proton magnetic resonance spectroscopy: a phantom study at 4 Tesla. J Magn Reson 208: 210-218.

Kakeda S, Korogi Y, Moriya J, Ohnari N, Sato T, Ueno S et al (2011). Influence of work shift on glutamic acid and gammaaminobutyric acid (GABA): evaluation with proton magnetic resonance spectroscopy at 3T. Psychiatry Res 192: 55-59.

Karolewicz B, Maciag D, O'Dwyer G, Stockmeier CA, Rajkowska G (2010). Reduced Level of Glutamic Acid Decarboxylase-67 kDa in the Prefrontal Cortex in Major Depression. Int J Neuropsychopharmacol 13: 411-420.

Klumpers UM, Veltman DJ, Drent ML, Boellaard R, Comans EF, Meynen $\mathrm{G}$ et al (2010). Reduced parahippocampal and lateral temporal GABAA-[11C]flumazenil binding in major depression: preliminary results. Eur J Nucl Med Mol Imaging 37: 565-574.

Lefaucheur JP, Lucas B, Andraud F, Hogrel JY, Bellivier F, Del Cul $A$ et al (2008). Inter-hemispheric asymmetry of motor corticospinal excitability in major depression studied by transcranial magnetic stimulation. J Psychiatr Res 42: 389-398.

Levinson AJ, Fitzgerald PB, Favallli G, Blumberger DM, Daigle M, Daskalakis ZJ (2010). Evidence of cortical inhibitory deficits in major depressive disorder. Biol Psychiatry 67: 458-464.

Licata SC, Jensen JE, Penetar DM, Prescot AP, Lukas SE, Renshaw $P F$ (2009). A therapeutic dose of zolpidem reduces thalamic GABA in healthy volunteers: a proton MRS study at $4 \mathrm{~T}$. Psychopharmacology 203: 819-829.

Lin Y, Stephenson MC, Wharton SJ, Xin L, Mougin OE, Napolitano A et al (2011). Investigating the metabolic changes due to visual stimulation using functional proton magnetic resonance spectroscopy at 7T. Proc Intl Soc Mag Reson Med 19: 311.
Littner M, Hirshkowitz M, Kramer M, Kapen S, Anderson WM, Bailey D et al (2003). Practice parameters for using polysomnography to evaluate insomnia: an update. Sleep 26: 754-760.

Luscher B, Shen Q, Sahir N (2011). The GABAergic deficit hypothesis of major depressive disorder. Mol Psychiatry 16: 383-406.

Maciag D, Hughes J, O'Dwyer G, Pride Y, Stockmeier CA, Sanacora $\mathrm{G}$ et al (2010). Reduced density of calbindin immunoreactive GABAergic neurons in the occipital cortex in major depression: relevance to neuroimaging studies. Biol Psychiatry 67: 465-470.

Mescher M, Merkle H, Kirsch J, Garwood M, Gruetter R (1998). Simulataneous in vivo spectral editing and water suppression. NMR Biomed 11: 266-272.

Montagna P (2005). Fatal familial insomnia: a model disease in sleep physiopathology. Sleep Med Rev 9: 339-353.

Morin CM (1993). Insomnia: Psychological Assessment and Management. Guilford Press: New York.

Morin CM, Vallières A, Ivers H (2007). Dysfunctional beliefs and attitudes about sleep (DBAS): validation of a brief version (DBAS-16). Sleep 30: 1547-1554.

Neckelmann D, Mykletun A, Dahl AA (2007). Chronic insomnia as a risk factor for developing anxiety and depression. Sleep 30: 873-880.

Nierenberg AA, Husain MM, Trivedi MH, Fava M, Warden D, Wisniewski SR et al (2010). Residual symptoms after remission of major depressive disorder with citalopram and risk of relapse: a STAR ${ }^{\star D}$ report. Psychol Med 40: 41-50.

Nofzinger EA, Buysse DJ, Germain A, Price JC, Miewald JM, Kupfer DJ (2004). Functional neuroimaging evidence for hyperarousal in insomnia. Am J Psychiatry 161: 2126-2128.

Nofzinger EA, Nissen C, Germain A, Moul D, Hall M, Price JC et al (2006). Regional cerebral metabolic correlates of WASO during NREM sleep in insomnia. J Clin Sleep Med 2: 316-322.

Ohayon MM, Roth T (2003). Place of chronic insomnia in the course of depressive and anxiety disorders. J Psychiatr Res 37: 9-15.

Périco CA, Skaf CR, Yamada A, Duran F, Buchpiguel CA, Castro CC et al (2005). Relationship between regional cerebral blood flow and separate symptom clusters of major depression: a single photon emission computed tomography study using statistical parametric mapping. Neurosci Lett 384: 265-270.

Petty F, Schlesser MA (1981). Plasma GABA in affective illness. A preliminary investigation. J Affect Disord 3: 339-343.

Price RB, Shungu DC, Mao X, Nestadt P, Kelly C, Collins KA et al (2009). Amino acid neurotransmitters assessed by proton magnetic resonance spectroscopy: relationship to treatment resistance in major depressive disorder. Biol Psychiatry 65: 792-800.

Provencher SW (1993). Estimation of metabolite concentrations from localized in vivo proton NMR spectra. Magn Reson Med 30: 672-679.

Provencher SW (2001). Automatic quantitation of localized in vivo 1H spectra with LCModel. NMR Biomed 14: 260-264.

Rajkowska G, O’Dwyer G, Teleki Z, Stockmeier CA, MiguelHidalgo JJ (2007). GABAergic neurons immunoreactive for calcium binding proteins are reduced in the prefrontal cortex in major depression. Neuropsychopharmacology 32: 471-482.

Riemann D, Spiegelhalder K, Feige B, Voderholzer U, Berger M, Perlis $M$ et al (2010). The hyperarousal model of insomnia: a review of the concept and its evidence. Sleep Med Rev 14: 19-31.

Sanacora G (2010). Cortical inhibition, gamma-aminobutyric acid, and major depression: there is plenty of smoke but is there fire? Biol Psychiatry 67: 397-398.

Sanacora G, Gueorguieva R, Epperson CN, Wu YT, Appel M, Rothman DL et al (2004). Subtype-specific alterations of gammaaminobutyric acid and glutamate in patients with major depression. Arch Gen Psychiatry 61: 705-713.

Sanacora G, Mason GF, Rothman DL, Behar KL, Hyder F, Petroff OA et al (1999). Reduced cortical gamma-aminobutyric acid 
levels in depressed patients determined by proton magnetic resonance spectroscopy. Arch Gen Psychiatry 56: 1043-1047.

Sanacora G, Mason GF, Rothman DL, Hyder F, Ciarcia JJ, Ostroff $\mathrm{RB}$ et al (2003). Increased cortical GABA concentrations in depressed patients receiving ECT. Am J Psychiatry 160: 577-579.

Saper C, Scammell T, Lu J (2005). Hypothalamic regulation of sleep and circadian rhythms. Nature 437: 1257-1263.

Simon GE, VonKorff M (1997). Prevalence, burden, and treatment of insomnia in primary care. Am J Psychiatry 154: 1417-1423.

Smith MT, Perlis ML, Chengazi VU, Pennington J, Soeffing J, Ryan JM et al (2002). Neuroimaging of NREM sleep in primary insomnia: a Tc-99-HMPAO single photon emission computed tomography study. Sleep 25: 325-335.

Steriade M (2003). The corticothalamic system in sleep. Front Biosci 8: D878-D899.
Szklo-Coxe M, Young T, Peppard PE, Finn LA, Benca RM (2010). Prospective associations of insomnia markers and symptoms with depression. Am J Epidemiol 171: 709-720.

Villablanca J, Salinas-Zeballos M (1972). Sleep-wakefulness, EEG and behavioral studies of chronic cats without the thalamus-the 'athalamic cat. Arch Ital Biol 110: 383-411.

Weissman MM, Greenwald S, Nino-Murcia G, Dement WC (1997). The morbidity of insomnia uncomplicated by psychiatric disorders. Gen Hosp Psychiatry 19: 245-250.

Winkelman JW, Buxton OM, Jensen JE, Benson KL, O'Connor SP, Wang W et al (2008). Reduced brain GABA in primary insomnia: preliminary data from $4 \mathrm{~T}$ proton magnetic resonance spectroscopy (1H-MRS). Sleep 31: 1499-1506.

Winkelman JW, Finn L, Young T (2006). Prevalence and correlates of restless legs syndrome symptoms in the Wisconsin Sleep Cohort. Sleep Med 7: 545-552. 